\title{
Burden of Atopic Dermatitis in the United States: Analysis of Healthcare Claims Data in the Commercial, Medicare, and Medi-Cal Databases
}

\author{
Sulena Shrestha - Raymond Miao - Li Wang · Jingdong Chao • \\ Huseyin Yuce $\cdot$ Wenhui Wei
}

Received: April 26, 2017 / Published online: July 13, 2017

(C) The Author(s) 2017. This article is an open access publication

\begin{abstract}
Introduction: Comparative data on the burden of atopic dermatitis (AD) in adults relative to the general population are limited. We performed a large-scale evaluation of the burden of disease among US adults with $\mathrm{AD}$ relative to matched non-AD controls, encompassing comorbidities, healthcare resource utilization (HCRU), and costs, using healthcare claims data. The impact of AD disease severity on these outcomes was also evaluated.

Methods: Adult AD patients in the Commercial $(n=83,106), \quad$ Medicare $\quad(n=31,060), \quad$ and

Enhanced content To view enhanced content for this article go to http://www.medengine.com/Redeem/1AD 8F060712D4B1C.

Electronic supplementary material The online version of this article (doi:10.1007/s12325-017-0582-z) contains supplementary material, which is available to authorized users.
\end{abstract}

S. Shrestha $\cdot$ L. Wang $(\square)$

STATinMED Research/SIMR, Inc., Plano, TX, USA e-mail: lwang@statinmed.com

R. Miao

Sanofi US, Bridgewater, NJ, USA

J. Chao · W. Wei

Regeneron Pharmaceuticals, Inc., Tarrytown, NJ, USA

H. Yuce

New York City College of Technology-CUNY, Brooklyn, NY, USA
Medi-Cal $(n=5550)$ databases were matched $(1: 1)$ to non-AD controls by demographic characteristics. AD patients were stratified by disease severity (higher, lower) using treatment as a surrogate measure of severity. The comorbidity burden, HCRU, and costs were evaluated during a 12-month follow-up period.

Results: In the Commercial, Medicare, and Medi-Cal populations, patients with AD had a significantly higher overall comorbidity burden $(P<0.0001)$, an increased risk of asthma and allergic rhinitis (both $P<0.0001$ ), higher HCRU $(P<0.05)$, and higher mean total per patient costs (Commercial: US $\$ 10,461$ versus US $\$ 7187$; Medicare: US\$16,914 versus US\$13,714; MediCal; US $\$ 19,462$ versus US\$10,408; all $P<0.0001)$, compared with matched non-AD controls. Higher disease severity was associated with an increased comorbidity burden $(P<0.0001)$, HCRU $(P<0.05)$, and total costs (Commercial: US\$14,580 versus US\$7192; Medicare: US\$21,779 versus US\$12,490; MediCal; US\$22,123 versus US\$16,639; all $P<0.0001)$ relative to lower severity disease.

Conclusion: In this large-scale, healthcare claims database analysis, AD patients had a significantly higher comorbidity burden, HCRU, and costs compared with matched non-AD controls. Higher disease severity was associated with an even greater comorbidity and economic burden.

Funding: Sanofi and Regeneron Pharmaceuticals, Inc. 
Keywords: Atopic dermatitis; Burden of disease; Comorbidities; Dermatology; Disease severity; Healthcare resource utilization; Insurance claims database

\section{INTRODUCTION}

Atopic dermatitis (AD) is a chronic skin disorder, characterized by immune-mediated inflammation, intense itching, and eczematous lesions [1]. In most cases, $\mathrm{AD}$ first occurs in infants and children [2,3], and while the disease often resolves by puberty $[3,4]$, in about $50 \%$ of patients $\mathrm{AD}$ persists into adulthood and becomes a chronic, lifelong condition $[5,6]$. However, $\mathrm{AD}$ can also present for the first time in adults (adult-onset AD) [7-9]. The estimated overall lifetime prevalence of $\mathrm{AD}$ in US adults is $3.2 \%[10]$.

Topical corticosteroids are the established first-line anti-inflammatory treatment for $\mathrm{AD}$; however, calcineurin inhibitors, non-steroidal anti-inflammatory agents, are an alternative topical treatment option [11-13]. Adult patients with moderate-to-severe disease (20-46\% of patients [14-18]), whose symptoms are generally not well controlled with topical therapy, can be treated with short-term (4-8 weeks) phototherapy in the form of ultraviolet radiation [13]. In the event that topicals and/or phototherapy fail to control the disorder, systemic immunosuppressants (cyclosporine, azathioprine, methotrexate, or mycophenolate mofetil) are prescribed. However, the efficacy of these agents is based on inconsistent supporting evidence from clinical trials; moreover, they are associated with a substantial risk of severe adverse effects [11-13]. Thus, immunosuppressants are not approved for the treatment of $\mathrm{AD}$ in the USA and are typically used off-label. Systemic corticosteroids are also used for rescue treatment of moderate-to-severe $\mathrm{AD}$ patients despite limited high-quality evidence supporting the efficacy and safety of these agents in the treatment of AD [11-13, 19].

Several studies have shown that adult AD is associated with a substantial patient burden, including an increased prevalence of atopic comorbidities (asthma, nasal, and food allergies) and neuropsychiatric conditions (anxiety, depression, sleep disturbance), as well as reductions in work productivity and health-related quality of life [20-24]. However, there remains a lack of comparative data on the burden of $\mathrm{AD}$ in adult patients relative to the general population in the USA. To that end, the primary objective of this analysis was to undertake a large-scale evaluation of the disease burden among US adults with AD relative to matched non-AD controls, encompassing comorbidities, healthcare resource utilization (HCRU), and healthcare costs. The secondary objective was to evaluate the impact of AD disease severity on these outcomes.

\section{METHODS}

\section{Data Sources}

Patient-level data were extracted from the Optum $^{\circledR}$ (Eden Prairie, MN, USA) Commercial and Medicare databases, and the Medi-Cal database. The Commercial and Medicare insurance claims databases contain patient demographic information, inpatient admission records, use of outpatient services and prescription drugs, and costs of services. Medi-Cal contains patient demographics, information on inpatient and outpatient care, and prescription drug usage for low-income and disabled California residents. In compliance with the 1996 Health Insurance Portability and Accountability Act, the databases consist of fully de-identified datasets, to protect the identities of both patients and data contributors.

The study conformed with the Helsinki Declaration of 1964, as revised in 2013, concerning human and animal rights; and Springer's policy concerning informed consent has been followed.

\section{Study Design}

\section{Commercial and Medicare Data}

The study period ranged from January 1, 2010 through September 30, 2015. For each AD patient, the first physician diagnosis of $\mathrm{AD}$ 
(ICD-9-CM code 691.8) during the identification period was the index event, and the date of this first diagnostic claim was defined as the index date. The index event identification period ranged from June 30, 2010 through September 30, 2014, and patient data were assessed until the end of the study (Fig. 1). The baseline period was defined as the 6-month period prior to the index date; the follow-up period was defined as the 12-month period after the index date.

\section{Medi-Cal Data}

The study period ranged from January 1, 2010 through December 31, 2014. The index event identification period ranged from June 30, 2010 through December 31, 2013. The baseline and follow-up periods were as described for the Commercial and Medicare data (Fig. 1).

\section{Analysis Populations}

\section{AD Cohort}

Adult AD patients [Commercial: $\geq 18$ years; Medicare: $\geq 65$ years; Medi-Cal: $18-64$ years (on the index date)] were included if they had at least one diagnostic medical claim for AD during the index event identification period, and continuous medical and pharmacy enrollment during the 6-month baseline period through the 12-month follow-up period. Patients with missing values for sex, age, race, or region (Commercial and Medicare) were excluded.

\section{AD Severity Subgroups}

$\mathrm{AD}$ patients were stratified by treatment, which was used as a surrogate measure of severity because of the lack of clinical assessment of disease severity in these databases. The higher disease severity subgroup comprised patients who had at least one medical or pharmacy claim for systemic corticosteroids, systemic immunosuppressants, or phototherapy during the 12-month follow-up period. The lower disease severity subgroup comprised patients who did not receive any claims for systemic corticosteroids, systemic immunosuppressants, or phototherapy, and had only received topical corticosteroids or topical calcineurin inhibitors, or had no treatment for $\mathrm{AD}$ during the 12-month follow-up period.

\section{Non-AD Control Cohort}

Adults with no medical diagnosis of AD during the entire study period were randomly assigned the index date within the index event identification period. Those with continuous medical and pharmacy enrollment during the 6-month baseline and 12-month follow-up period were included in the non-AD control cohort. Patients with missing values for sex, age, race, or region (Commercial and Medicare) were excluded.

\section{Matching of AD Patients and Non-AD Controls} $\mathrm{AD}$ patients were matched (1:1) with non-AD control patients by demographic characteristics [age, sex, race, region (Commercial and

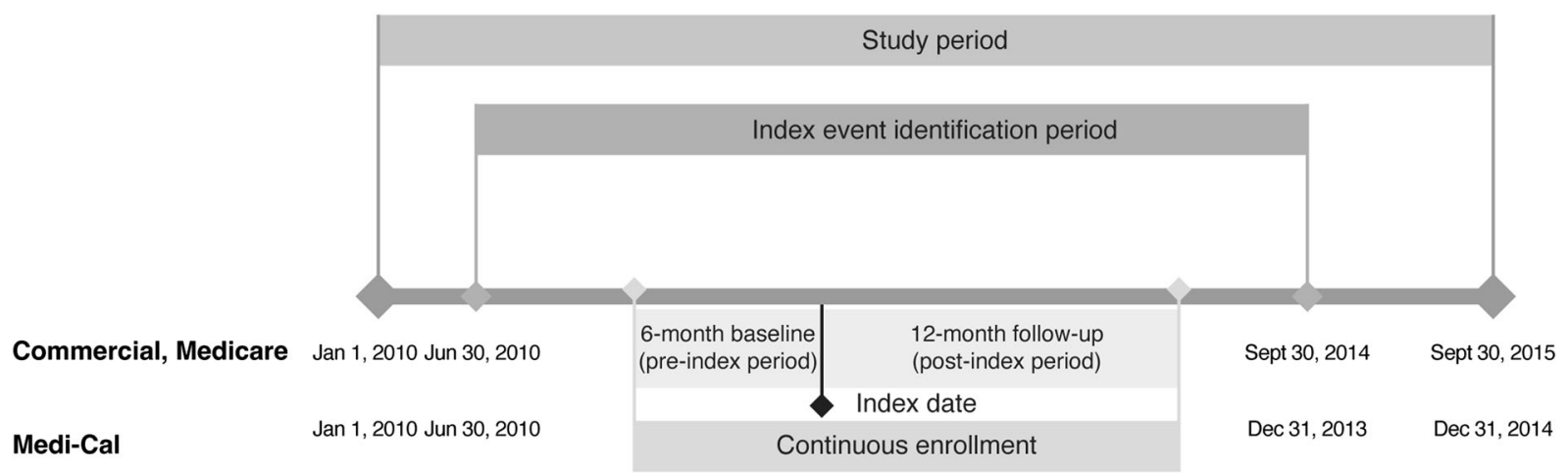

Fig. 1 Sample selection in the Commercial, Medicare, and Medi-Cal populations. For atopic dermatitis (AD) patients, the index date was defined as the date of the first diagnostic claim of $\mathrm{AD}$; for non- $\mathrm{AD}$ controls, it was randomly assigned within the index event identification period 
Medicare); age, sex, race (Medi-Cal)] and index year. If more than one control patient matched a particular $\mathrm{AD}$ patient, then one of the matched controls was randomly selected to be included in the matched analysis population. If no matched control patient was available, then the AD patient was excluded from the matched analysis population.

\section{Comorbidities}

Comorbid variables were evaluated during the 12-month follow-up period. Overall comorbidity burden was measured using the Deyo-modified Charlson Comorbidity Index (CCI) score calculated by assigning a weight ranging from 1 to 6 based on the adjusted risk of mortality for each of 17 conditions. The sum of the weights results in an overall comorbidity score, with higher scores indicating higher risks of mortality [25-27]. In addition, the prevalence of the following 18 prespecified comorbidities were measured, including "atopic march" conditions (asthma, allergic rhinitis, food allergy); other respiratory conditions (chronic pulmonary disease, chronic rhinosinusitis), other AD-related conditions (allergic urticaria, autoimmune disorders, conjunctivitis, esophagitis, nasal polyps); infections (bacterial, viral, and fungal infections); psychological conditions (attention deficit hyperactivity disorder, anxiety, depression, sleep disorders), and obesity. These conditions have been previously shown to be associated with $\mathrm{AD}[28,29]$.

\section{Healthcare Resource Utilization}

All-cause HCRU was evaluated during the 12-month follow-up period for the following categories: inpatient visits, emergency room (ER) visits, outpatient visits, pharmacy prescriptions, and specialist (allergist/dermatologist) visits.

\section{Healthcare Costs}

Direct healthcare costs were evaluated during the 12-month follow-up for the following categories: outpatient costs, inpatient stay costs, pharmacy costs, ER costs, and total costs (outpatient + inpatient + pharmacy). Only costs incurred by payers were included in the analyses; patient co-payments and non-allowed charges were excluded. Costs were adjusted to 2014 prices for Medi-Cal data and 2015 prices for Commercial and Medicare data using the annual medical care component and drug costs component of the Consumer Price Index to reflect inflation.

\section{Statistical Analyses}

\section{Comorbidities}

The burden of comorbidities was modeled using logistic regression, including the calculation of odds ratios (ORs) with 95\% confidence intervals (CIs). The dependent variable was the presence/ absence of comorbidities; independent variables included patient demographic characteristics and non-AD-related comorbidities.

\section{Healthcare Resource Utilization}

HCRU data were modeled using logistic regression. In particular, conditional logistic regression was performed among 1:1 matched $\mathrm{AD}$ and non-AD cohorts. Dependent variables included binary variables such as any inpatient, outpatient, ER, and pharmacy visits; independent variables included patient demographic characteristics and non-AD-related comorbidities. Count variables were analyzed with negative binomial regression. To account for correlation between matched pairs, a generalized estimating equation was used.

\section{Costs}

To estimate adjusted cost differences between matched cohorts and disease severity groups, multivariate two-part regression models were used. Specifically, the estimated cost was calculated by multiplying the probability of having a nonzero cost (first part of the model) by the predicted nonzero cost values (second part of the model). In these models, the dependent variables were inpatient costs, outpatient costs, ER costs, pharmacy costs, and total costs; independent variables included all patients' demographic characteristics and non-AD-related comorbidities. 


\section{RESULTS}

\section{Analysis Populations}

Flowcharts of adult $\mathrm{AD}$ patients and non-AD controls, and higher and lower severity $\mathrm{AD}$ patients are shown in Fig. 2. After 1:1 matching of $\mathrm{AD}$ patients to non-AD controls, the Commercial AD cohort comprised 83,106 patients (higher severity, 35.7\%; lower severity, 64.3\%); the Medicare AD cohort 31,060 patients (higher severity, 40.4\%; lower severity, 59.6\%); and the Medi-Cal AD cohort 5550 patients (higher severity, $21.6 \%$; lower severity, $78.4 \%$ ).

\section{Baseline Demographics}

Given that matching was based on patient demographics, baseline demographic characteristics were identical between the matched $\mathrm{AD}$ a
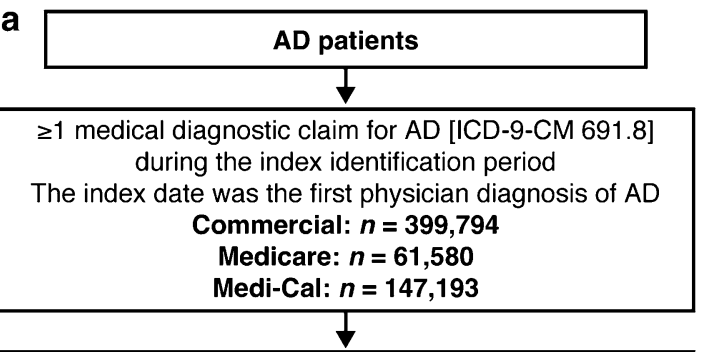

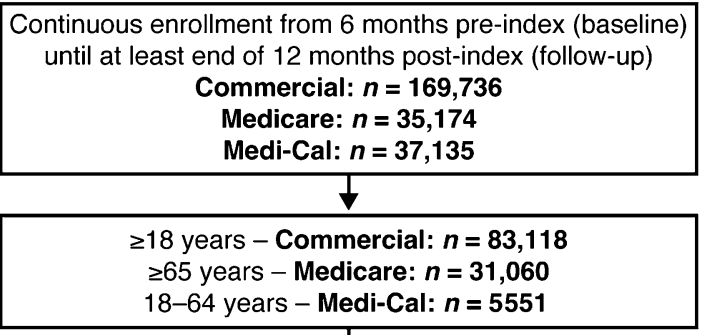
$\downarrow$

AD patients matched (1:1) to non-AD controls by identical demographic characteristics (age, gender, race, region [Commercial and Medicare]; age, gender, race [Medi-Cal])

Commercial: $n=83,106$

Medicare: $n=31,060$

Medi-Cal: $\boldsymbol{n}=\mathbf{5 5 5 0}$

Commercial: $n=83,106$

Medicare: $n=31,060$

Medi-Cal: $n=5550$

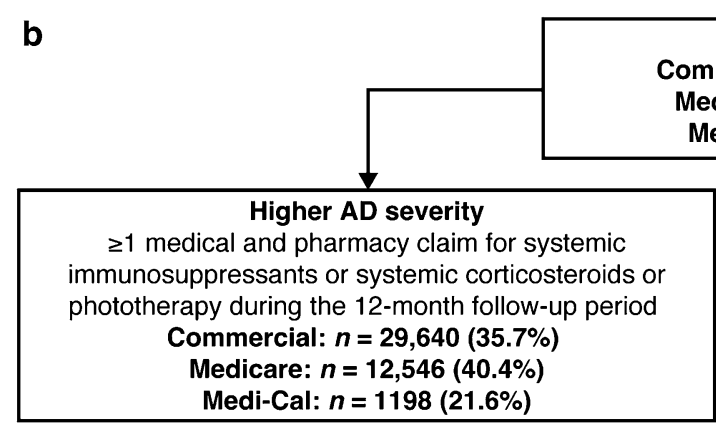

AD patients ommercial: $n=83,106$

Medicare: $n=31,060$

Medi-Cal: $\boldsymbol{n}=\mathbf{5 5 5 0}$

$\geq 1$ medical and pharmacy claim for systemic immunosuppressants or systemic corticosteroids or

Commercial: $\boldsymbol{n}=\mathbf{2 9}, \mathbf{6 4 0}(\mathbf{3 5 . 7} \%)$

Medi-Cal: $n=1198(21.6 \%)$

Fig. 2 Flowcharts of: a atopic dermatitis (AD) patients and matched non-AD controls; $\mathbf{b}$ AD patients stratified by disease severity 
and non-AD controls across the three insurance populations (Table 1). Baseline demographics were similar for $\mathrm{AD}$ patients with higher and lower disease severity (Table 2).

\section{Treatment Patterns}

Treatment pattern data for $\mathrm{AD}$ patients in the Commercial, Medicare, and Medi-Cal insurance cohorts is shown in Table 3. In line with current guidelines, the majority of $\mathrm{AD}$ patients (60-72\%) were treated with topical corticosteroids; however, $24-26 \%$ of patients received no treatment of any kind. With regard to systemic therapy, $21-40 \%$ of patients were treated with systemic corticosteroids, and approximately $1 \%$ received systemic immunosuppressants, with methotrexate accounting for $50-90 \%$ of immunosuppressant use.

\section{Comorbidity Burden}

Compared with non-AD controls, patients with $\mathrm{AD}$ had a significantly higher overall comorbidity burden in all three insurance populations, as indicated by mean (SD) Deyo-modified CCI scores (Supplementary Table 1): Commercial, 0.46 (1.10) versus 0.34 (0.98); Medicare, 1.92 (2.21) versus 1.75 (2.21); Medi-Cal, 0.78 (1.48) versus 0.52 (1.37) (all $P<0.0001$ ). AD patients with higher disease severity had an even larger comorbidity burden relative to lower severity patients (Supplementary Table 2): Commercial, 0.63 (1.28) versus 0.37 (0.97); Medicare, 2.18 (2.33) versus 1.75 (2.10); MediCal, $1.23 \quad(1.77)$ versus $0.66 \quad$ (1.36) (all $P<0.0001)$.

Figure 3 shows adjusted ORs for prespecified AD-related comorbidities in $\mathrm{AD}$ patients relative to matched non-AD controls. In the

Table 1 Baseline demographics of matched atopic dermatitis (AD) patients and non-AD controls

\begin{tabular}{|c|c|c|c|c|c|c|}
\hline & \multicolumn{2}{|l|}{ Commercial } & \multicolumn{2}{|l|}{ Medicare } & \multicolumn{2}{|l|}{ Medi-Cal } \\
\hline & $\begin{array}{l}\text { AD } \\
(n=83,106)\end{array}$ & $\begin{array}{l}\text { Non-AD } \\
(n=83,106)\end{array}$ & $\begin{array}{l}\mathrm{AD} \\
(n=31,060)\end{array}$ & $\begin{array}{l}\text { Non-AD } \\
(n=31,060)\end{array}$ & $\begin{array}{l}\mathrm{AD} \\
(n=5550)\end{array}$ & $\begin{array}{l}\text { Non-AD } \\
(n=5550)\end{array}$ \\
\hline $\begin{array}{l}\text { Age, years, mean } \\
\quad(\mathrm{SD})\end{array}$ & $43(14.6)$ & & $75(6.7)$ & & $37(13.7)$ & \\
\hline Female, $n(\%)$ & $51,390(61.8)$ & & $18,391(59.2)$ & & $4133(74.5)$ & \\
\hline \multicolumn{7}{|l|}{ Race, $n(\%)$} \\
\hline $\begin{array}{l}\text { African } \\
\text { American }\end{array}$ & $8847(10.7)$ & & $2247(7.2)$ & & $916(16.5)$ & \\
\hline Hispanic & $8231(9.9)$ & & $3329(10.7)$ & & $2054(37.0)$ & \\
\hline White & $55,652(67.0)$ & & $22,401(72.1)$ & & $1252(22.6)$ & \\
\hline Asian & $5777(7.0)$ & & $1630(5.3)$ & & $1129(20.3)$ & \\
\hline Other & $4599(5.5)$ & & $1453(4.7)$ & & $199(3.6)$ & \\
\hline \multicolumn{7}{|l|}{ US region } \\
\hline Northeast & $11,097(13.4)$ & & $4414(14.2)$ & & - & \\
\hline Midwest & $16,136(16.4)$ & & $5120(16.5)$ & & - & \\
\hline South & $38,671(46.5)$ & & $9582(30.9)$ & & - & \\
\hline West & $17,166(20.7)$ & & $11,332(36.5)$ & & $5550(100.0)^{\mathrm{a}}$ & \\
\hline
\end{tabular}

$\mathrm{AD}$ patients and non-AD controls were matched (1:1) by demographic characteristics

a The Medi-Cal database contains information about Californian residents only 
Table 2 Baseline demographics of atopic dermatitis patients stratified by disease severity

\begin{tabular}{|c|c|c|c|c|c|c|}
\hline & \multicolumn{2}{|c|}{ Commercial $(n=83,106)$} & \multicolumn{2}{|c|}{ Medicare $(n=31,060)$} & \multicolumn{2}{|c|}{ Medi-Cal $(n=5550)$} \\
\hline & $\begin{array}{l}\text { Higher severity } \\
(n=29,640)\end{array}$ & $\begin{array}{l}\text { Lower severity } \\
(n=53,466)\end{array}$ & $\begin{array}{l}\text { Higher severity } \\
(n=12,546)\end{array}$ & $\begin{array}{l}\text { Lower severity } \\
(n=18,514)\end{array}$ & $\begin{array}{l}\text { Higher } \\
\text { severity } \\
(n=1198)\end{array}$ & $\begin{array}{l}\text { Lower } \\
\text { severity } \\
(n=4352)\end{array}$ \\
\hline $\begin{array}{l}\text { Age, years, } \\
\text { mean }(\mathrm{SD})\end{array}$ & $45(14.3)$ & $42(14.7)$ & $75(6.6)$ & $76(6.8)$ & $39(13.4)$ & $36(13.8)$ \\
\hline $\begin{array}{l}\text { Female, } \\
n(\%)\end{array}$ & $18,794(63.4)$ & $32,596(61.0)$ & $7637(60.9)$ & $10,754(58.1)$ & $930(77.6)$ & $3203(73.6)$ \\
\hline \multicolumn{7}{|l|}{ Race, $n(\%)$} \\
\hline $\begin{array}{l}\text { African } \\
\text { American }\end{array}$ & $3293(11.1)$ & $5554(10.4)$ & $959(7.6)$ & $1288(7.0)$ & $187(15.6)$ & $729(16.8)$ \\
\hline Hispanic & $2932(9.9)$ & $5299(9.9)$ & $1396(11.1)$ & $1933(10.4)$ & $432(36.1)$ & $1622(37.3)$ \\
\hline White & $20,435(68.9)$ & $35,217(65.9)$ & $9076(72.3)$ & $13,325(72.0)$ & $328(27.4)$ & $924(21.2)$ \\
\hline Asian & $1462(4.9)$ & $4315(8.1)$ & $569(4.5)$ & $1061(5.7)$ & $211(17.6)$ & $918(21.1)$ \\
\hline Other & $1518(5.1)$ & $3081(5.8)$ & $546(4.4)$ & $907(4.9)$ & $40(3.3)$ & $159(3.7)$ \\
\hline \multicolumn{7}{|l|}{ Region, $n(\%)$} \\
\hline Northeast & $3160(10.7)$ & $7937(14.8)$ & $1516(12.1)$ & $2898(15.7)$ & - & - \\
\hline Midwest & $5953(20.1)$ & $10,183(19.1)$ & $1973(15.7)$ & $3147(17.0)$ & - & - \\
\hline South & $15,590(52.6)$ & $23,081(43.2)$ & $4511(36.0)$ & $5071(27.4)$ & - & - \\
\hline West & $4924(16.6)$ & $12,242(22.9)$ & $4328(34.5)$ & $7004(37.8)$ & $1198(100.0)^{\mathrm{a}}$ & $4352(100.0)^{\mathrm{a}}$ \\
\hline
\end{tabular}

a The Medi-Cal database contains information about Californian residents only

Commercial (Fig. 3a) and Medicare (Fig. 3b) populations, all 18 evaluated AD-related comorbid conditions were significantly more likely to occur in $\mathrm{AD}$ patients than in non-AD controls $(P<0.0001)$; in the Medi-Cal population (Fig. 3c), 16/18 conditions showed a significantly increased risk in in AD patients, with only nasal polyps and food allergy showing nonsignificant ORs. AD was associated with an increased risk of the atopic conditions asthma (Commercial: OR 2.51; Medicare: OR 1.65; Medi-Cal: OR 2.78; all $P<0.0001)$ and allergic rhinitis (OR 3.29, 2.34, 3.84, respectively; all $P<0.0001)$, the respiratory conditions chronic pulmonary disease (OR 2.07, 1.28, 2.40; all $P<0.0001$ ) and chronic rhinosinusitis (OR 1.65, $1.49,2.64$; all $P<0.0001)$, and the neuropsychiatric conditions sleep disorder (OR 1.77, 1.50, 2.93; all $P<0.0001)$ and anxiety (OR 1.48,
1.46, 1.83; all $P<0.0001)$. Other comorbid conditions with a significantly increased risk in $\mathrm{AD}$ patients in all three populations were allergic urticaria (OR 6.79, 7.71, 5.52; all $P<0.0001$ ) and conjunctivitis (OR 2.71, 2.15, and 3.00; all $P<0.0001)$.

Figure 4 shows adjusted ORs for AD-related comorbidities stratified by disease severity. Compared with lower AD severity patients, patients with higher AD severity had a significantly increased risk of asthma (Commercial: OR 2.32; Medicare: OR 2.44; Medi-Cal: OR 3.24; all $P<0.0001$ ), allergic rhinitis (OR 1.90, 1.77, 1.93, respectively; all $P<0.0001)$, chronic pulmonary disease (OR 2.32, 2.03, 2.74; all $P<0.0001$ ), and chronic rhinosinusitis (OR $2.29,1.84,1.90$; all $P<0.0001)$. Other comorbid conditions with a significantly increased risk in patients with higher versus lower disease 
Table 3 Treatment regimens of atopic dermatitis patients

\begin{tabular}{lccc}
\hline Treatment usage, $\boldsymbol{n}(\%)$ & Commercial $(\boldsymbol{n}=\mathbf{8 3 , 1 0 6})$ & Medicare $(\boldsymbol{n}=\mathbf{3 1 , 0 6 0})$ & Medi-Cal $(\boldsymbol{n}=\mathbf{5 5 5 0})$ \\
\hline No treatment & $21,271(25.6)$ & $8121(26.2)$ & $1306(23.5)$ \\
Phototherapy & $821(1.0)$ & $342(1.1)$ & $24(0.4)$ \\
Any systemic immunosuppressant & $892(1.1)$ & $403(1.3)$ & $51(0.9)$ \\
Azathioprine & $186(0.2)$ & $78(0.3)$ & $4(0.1)$ \\
Cyclosporine & $170(0.2)$ & $25(0.1)$ & $8(0.1)$ \\
Methotrexate & $447(0.5)$ & $281(0.9)$ & $29(0.5)$ \\
Mycophenolate mofetil & $169(0.2)$ & $47(0.2)$ & $13(0.2)$ \\
Any systemic corticosteroid & $28,986(34.9)$ & $12,291(39.6)$ & $1165(21.0)$ \\
Oral & $20,267(24.4)$ & $7226(23.3)$ & $976(17.6)$ \\
Injectable & $13,792(16.6)$ & $6738(21.7)$ & $260(4.7)$ \\
Any topical corticosteroid & $50,593(60.9)$ & $18,723(60.3)$ & $3988(71.9)$ \\
High strength & $11,340(13.6)$ & $4020(12.9)$ & $870(15.7)$ \\
Low strength & $9477(11.4)$ & $3131(10.3)$ & $1135(20.5)$ \\
Medium strength & $28,893(34.8)$ & $11,515(37.1)$ & $2835(51.1)$ \\
Very high strength & $14,659(17.6)$ & $5754(18.5)$ & $393(7.1)$ \\
Any topical calcineurin inhibitor & $3665(4.4)$ & $354(1.1)$ & $59(1.1)$ \\
Tacrolimus & $2098(2.5)$ & $207(0.7)$ & $37(0.7)$ \\
Pimecrolimus & $1687(2.0)$ & $160(0.5)$ & $24(0.4)$ \\
\hline
\end{tabular}

severity in all three populations were allergic urticaria (OR 2.89, 3.30, 3.98; all $P<0.0001$ ) and nasal polyps [OR 3.35, $2.39(P<0.0001$, both), $3.73(P=0.02)]$.

\section{Healthcare Resource Utilization}

In all three insurance populations, a significantly greater proportion of $\mathrm{AD}$ patients required ER visits, outpatient visits, and pharmacy prescriptions compared with non-AD controls (all $P<0.05$; Supplementary Fig. 1). A significantly greater proportion of higher severity patients required inpatient visits (Commercial, Medicare), ER visits (Commercial, Medicare, Medi-Cal), pharmacy prescriptions (Commercial, Medicare), and specialist (allergist/dermatologist) visits (Commercial, Medicare, Medi-Cal) compared with lower severity patients (all $P<0.05$; Supplementary Fig. 2).
Across all three populations, AD patients had a significantly higher mean annual number of ER visits, outpatient visits, and pharmacy prescriptions than non-AD controls (all $P<0.05$; Fig. 5). In the Commercial and Medicare populations, higher severity patients used significantly greater annual mean resources across all five evaluated categories (inpatient visits, ER visits, outpatient visits, pharmacy prescriptions, allergist/dermatologist visits) compared with lower severity patients (all $P<0.05$; Fig. 6).

\section{Healthcare Costs}

Adjusted mean annual total per-patient costs were significantly higher in AD patients compared with non-AD controls in all three populations (Commercial: US\$10,461 versus US\$7187; Medicare: US\$16,914 versus US\$13,714; Medi-Cal; US\$19,462 versus 


\section{a}

Allergic rhinitis
Asthma
Food allergy
Chronic pulmonary disease
Chronic rhinosinusitis
Allergic urticaria
Autoimmune disorders
Conjuctivitis
Esophagitis
Nasal polyps
Bacterial infections
Fungal infections
Viral infections
ADHD
Anxiety
Depression
Sleep disorder
Obesity

Higher risk in non-AD $\longleftarrow$ controls

\section{b}

Allergic rhinitis

Asthma

Food allergy

Chronic pulmonary disease

Chronic rhinosinusitis

Allergic urticaria

Autoimmune disorders

Conjuctivitis

Esophagitis

Nasal polyps

Bacterial infections

Fungal infections

Viral infections

ADHD

Anxiety

Depression

Sleep disorder

Obesity

Higher risk in non-AD controls

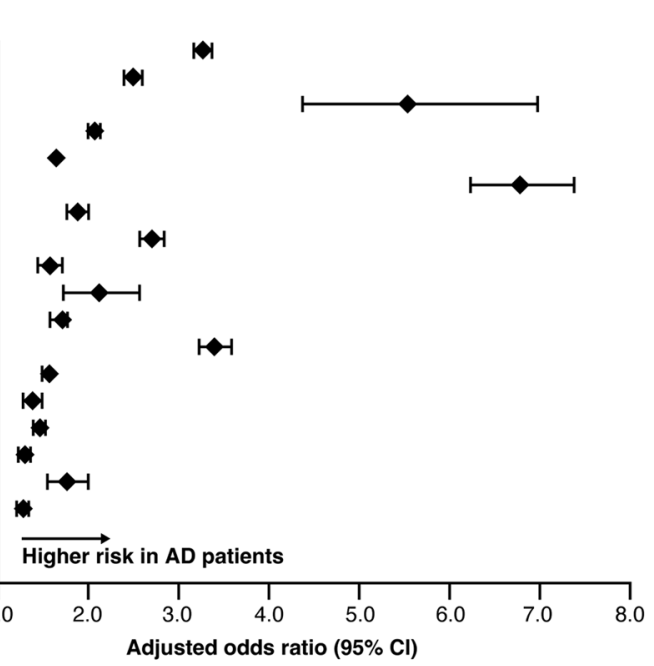

$<0.0001$

$<0.0001$

$<0.0001$

$<0.0001$

$<0.0001$

$<0.0001$

$<0.0001$

$<0.0001$

$<0.0001$

$<0.0001$

$<0.0001$

$<0.0001$

$<0.0001$

$<0.0001$

$<0.0001$

$<0.0001$

$<0.0001$

$<0.0001$

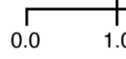

Adjusted odds ratio $(95 \% \mathrm{Cl})$

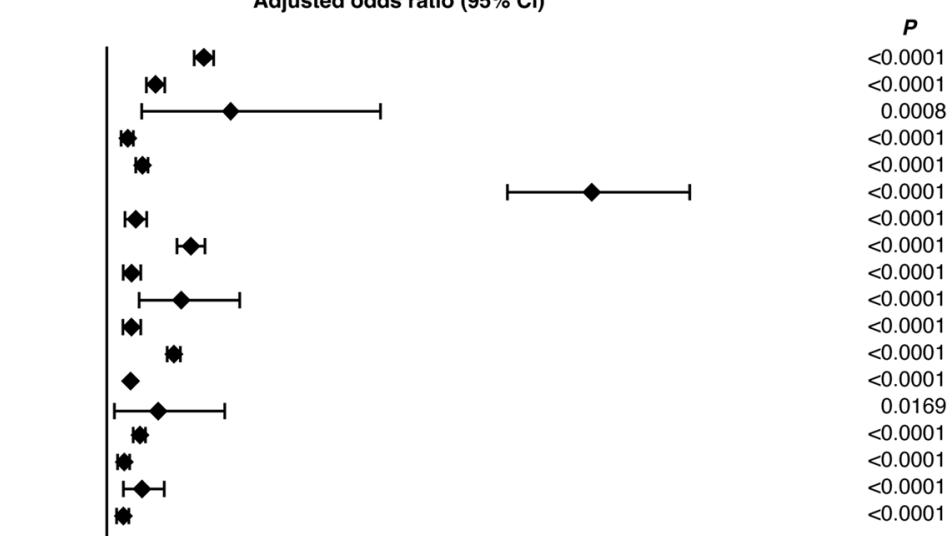

\section{C}

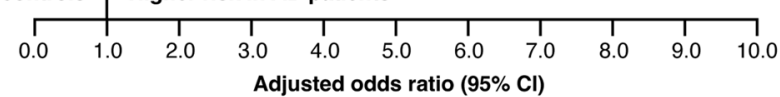

Allergic rhinitis

Asthma

Food allergy

Chronic pulmonary disease

Chronic rhinosinusitis

Allergic urticaria

Autoimmune disorders

Conjuctivitis

Esophagitis

Nasal polyps

Bacterial infections

Fungal infections

Viral infections

ADHD

Anxiety

Depression

Sleep disorder

Obesity

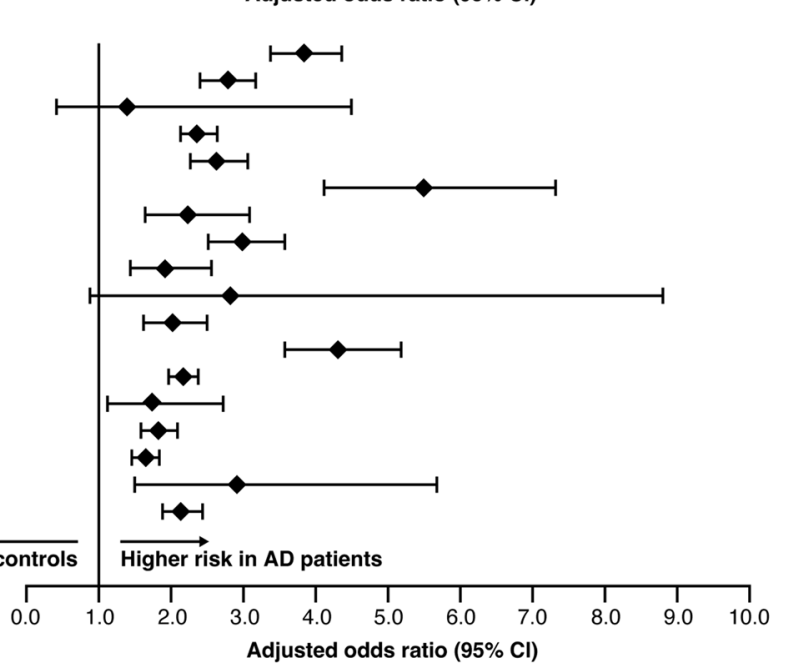

$<0.0001$

0.0731
-0.0001

$<0.0001$

$<0.0001$
0.0158

$<0.0001$

$<0.0001$

0.0016

$<0.0001$

Higher risk in non-AD controls

Higher risk in AD patients

Adjusted odds ratio $(95 \% \mathrm{Cl})$

Fig. 3 Adjusted odds ratios of prespecified comorbidities in atopic dermatitis $(\mathrm{AD})$ patients and matched non-AD controls in: a Commercial, b Medicare, and $\mathbf{c}$ Medi-Cal populations. Variables included in the generalized linear model: age, gender, race, and non-AD-related comorbidities. $A D H D$ attention deficit hyperactivity disorder, $C I$ confidence interval 
a

Allergic rhinitis
Asthma
Food allergy
Chronic pulmonary disease
Chronic rhinosinusitis
Allergic urticaria
Autoimmune disorders
Conjuctivitis
Esophagitis
Nasal polyps
Bacterial infections
Fungal infections
Viral infections
ADHD
Anxiety
Depression
Sleep disorder
Obesity

Higher risk in lower $A D \overleftarrow{\longleftarrow}$ severity

b

\begin{tabular}{rrrrrrr}
\hline & \multicolumn{1}{|c}{} & \multicolumn{1}{l}{} & \\
0.0 & 1.0 & 2.0 & 3.0 & 4.0 & 5.0
\end{tabular}

Allergic rhinitis
Asthma
Food allergy
Chronic pulmonary disease
Chronic rhinosinusitis
Allergic urticaria
Autoimmune disorders
Conjuctivitis
Esophagitis
Nasal polyps
Bacterial infections
Fungal infections
Viral infections
ADHD
Anxiety
Depression
Sleep disorder
Obesity

Adjusted odds ratio $(95 \% \mathrm{Cl})$

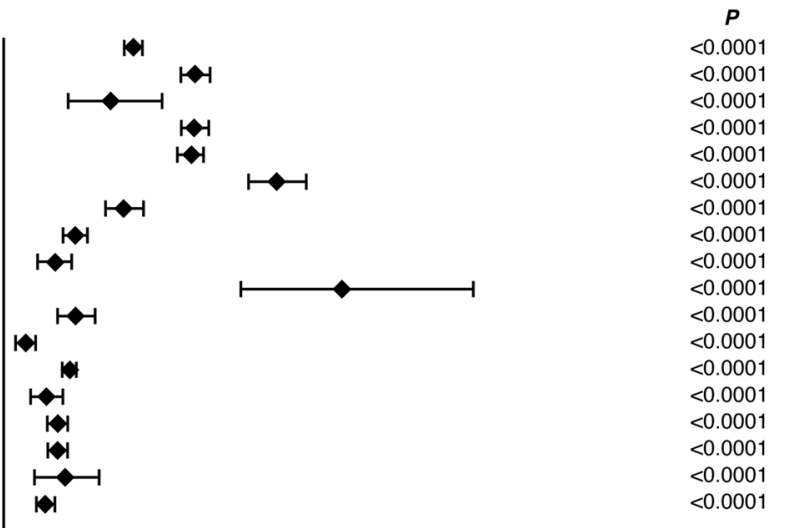

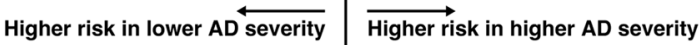

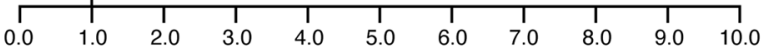

C

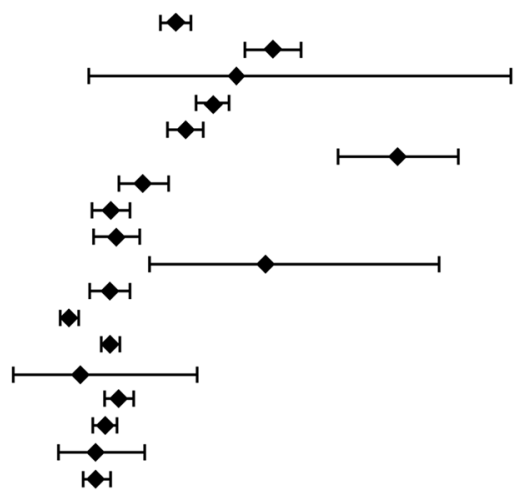

$P$

$<0.0001$

$<0.0001$

0.0137

$<0.0001$

$<0.0001$

$<0.0001$

$<0.0001$

$<0.0001$

$<0.0001$

$<0.0001$

$<0.0001$

0.3133

$<0.0001$

0.6967

$<0.0001$

$<0.0001$

0.1078

$<0.0001$

Adjusted odds ratio $(95 \% \mathrm{Cl})$

Allergic rhinitis

Asthma

Food allergy

Chronic pulmonary disease

Chronic rhinosinusitis

Allergic urticaria

Autoimmune disorders

Conjuctivitis

Esophagitis

Nasal polyps

Bacterial infections

Fungal infections

Viral infections

ADHD

Anxiety

Depression

Sleep disorder

Obesity

0.0783

0.0240

0.0535

0.7807

0.0002

0.0838

0.1033

0.0009

0.3738

0.0177

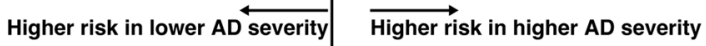

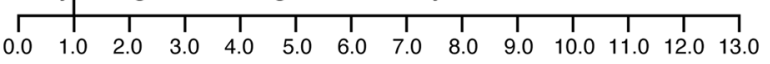

Adjusted odds ratio $(95 \% \mathrm{Cl})$

Fig. 4 Adjusted odds ratios of prespecified comorbidities stratified by disease severity in: a Commercial, b Medicare, and c Medi-Cal populations. Variables included in the generalized linear model: age, gender, race, and non-ADrelated comorbidities. $A D$ atopic dermatitis, $A D H D$ attention deficit hyperactivity disorder, $C I$ confidence interval 
US $\$ 10,408$; all $P<0.0001)$, mainly driven by outpatient costs and pharmacy prescription costs (Fig. 7). Corresponding total costs were also significantly increased for higher versus lower severity patients (Commercial: US\$14,580 versus US\$7192; Medicare: US\$21,779 versus US\$12,490; Medi-Cal; US\$22,123 versus US\$16,639; all $P<0.0001)$, again mainly driven by outpatient and prescription costs (Fig. 8).

\section{DISCUSSION}

In this retrospective healthcare claims database study, we performed a comparative analysis of the burden of disease in US adult AD patients relative to matched non-AD controls, encompassing comorbidities, HCRU, and healthcare costs. In addition, we examined the impact of AD disease severity on these outcomes. Data from approximately 240,000 patients in the Commercial, Medicare, and Medi-Cal insurance databases were analyzed, representing, to the best of our knowledge, the largest analysis to date of the clinical and economic burden associated with AD.

The results showed that relative to matched non-AD controls, adult patients with AD had a significantly higher overall comorbidity burden, and a significantly increased risk of atopic comorbidities, including allergic urticaria, allergic rhinitis, and asthma. In addition, there was a significantly increased risk of atopic conditions in patients with higher severity disease relative to lower severity disease. This association between $\mathrm{AD}$ and atopic comorbidities has been recognized in previous studies [3, 30, 31], and is consistent with the "atopic march" concept in which $\mathrm{AD}$ precedes the development of other allergic disorders [32].

In accordance with the high comorbidity burden, overall HCRU was significantly higher in AD patients compared with non-AD controls, and in higher severity patients compared with lower severity patients. The data show that the increased HCRU was primarily driven by outpatient visits and pharmacy prescriptions. A recent study of the burden of AD in US adults, based on data from the 2013 National Health and Wellness Survey (NHWS) [23], also found that HCRU was higher in
$\mathrm{AD}$ patients compared with non-AD controls. However, in the NHWS study, the increased HCRU was driven solely by outpatient visits as pharmacy utilization was substantially lower than that seen in the current analysis, with $48 \%$ of patients receiving prescriptions in the NHWS study compared with $92-99 \%$ in the current study. It should be noted that the NHWS study was based on self-reported data and it is possible that pharmacy utilization was under-reported.

The higher HCRU in AD patients relative to non-AD controls translated to annual mean incremental per-patient costs of US\$3200-3300 in the Commercial and Medicare populations, and around US\$9000 in the Medi-Cal population. To put these costs in perspective, one study reported that the estimated incremental per-patient costs incurred by US patients with psoriasis, another common, chronic skin disorder, are US\$2284 [33]. Overall, the cost data reported here are consistent with those of previous studies showing that $\mathrm{AD}$ is associated with substantial HCRU and healthcare costs [34-36], and that higher $\mathrm{AD}$ severity correlates with increased HCRU and costs [36, 37].

Given that the higher severity subgroup comprised patients treated with systemic therapy or phototherapy, it is reasonable to suggest that most of these patients had moderate-to-severe $\mathrm{AD}$. Thus, the cost difference between the higher severity subgroup and non-AD controls (Commercial: US\$7393; Medicare: US\$8065; Medi-Cal: US\$11,715) is a good approximation of moderate-to-severe AD-related costs. By applying the distribution of US insurance plans (Commercial: 60.9\%; Medicare: $17.6 \%$; Medicaid: $21.5 \%$ [38]) to these costs, the mean annual per-person costs incurred by patients with moderate-to-severe AD can be estimated at US\$8438. With 1.6 million uncontrolled moderate-to-severe $\mathrm{AD}$ adult patients in the USA $[10,18,39,40]$, the total healthcare costs of uncontrolled moderate-to-severe adult $\mathrm{AD}$ in the USA can be estimated at $\$ 13$ billion.

It can be argued that the cost difference between the higher and lower severity groups (Commercial: US\$7388; Medicare: US\$9289; Medi-Cal: US\$5484) could equate to a potential cost offset obtained by effectively treating 

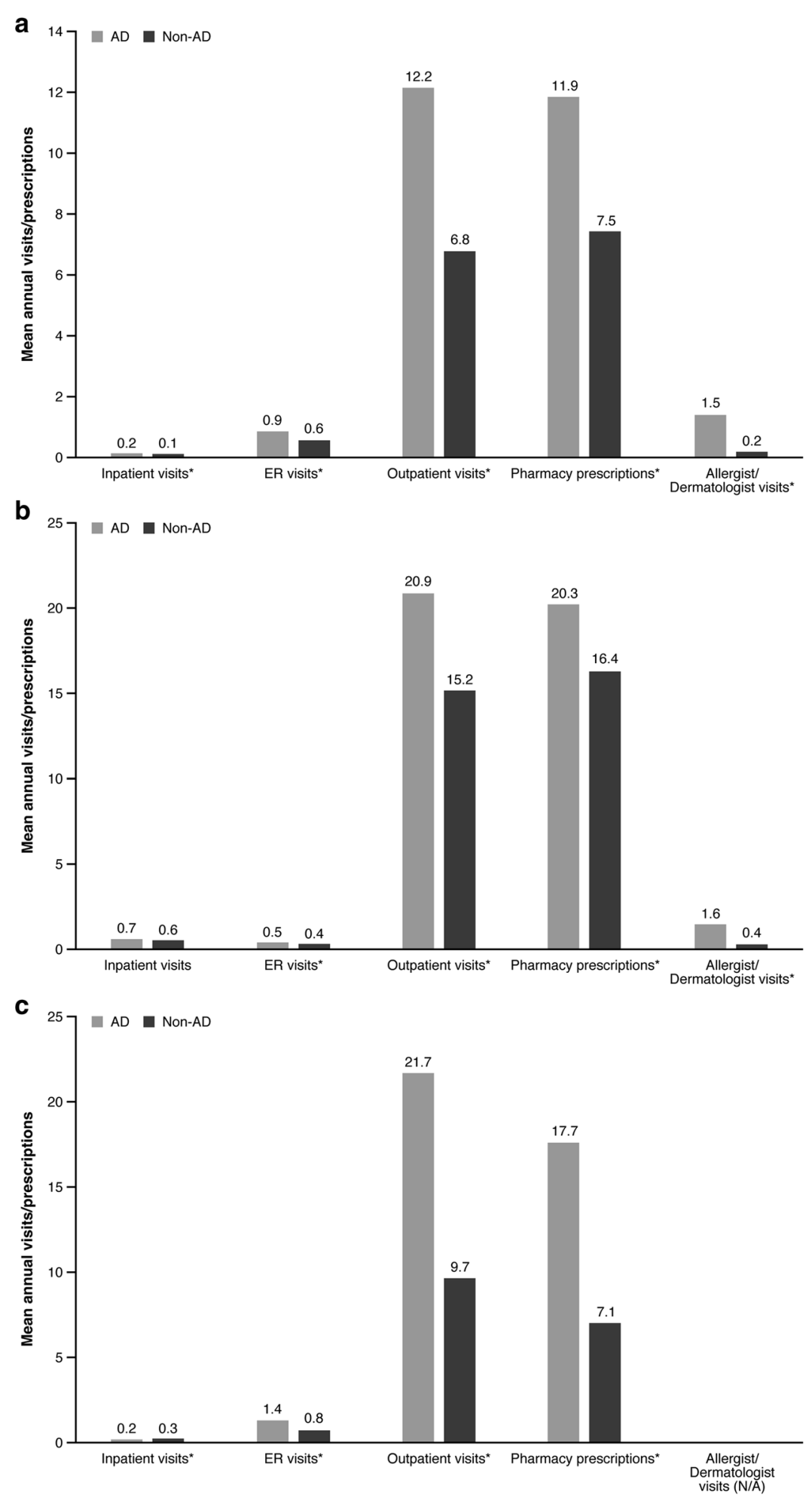

Fig. 5 Adjusted mean annual number of healthcare visits and number of prescriptions per patient in $\mathrm{AD}$ patients and matched non-AD controls in: a Commercial, b Medicare, and $\mathbf{c}$ Medi-Cal populations. ${ }^{*} P<0.05$. Variables included in the generalized linear model: age, gender, race, non-AD-related comorbidities, and region (Commercial and Medicare only). $A D$ atopic dermatitis, $E R$ emergency room, $N / A$ not applicable 

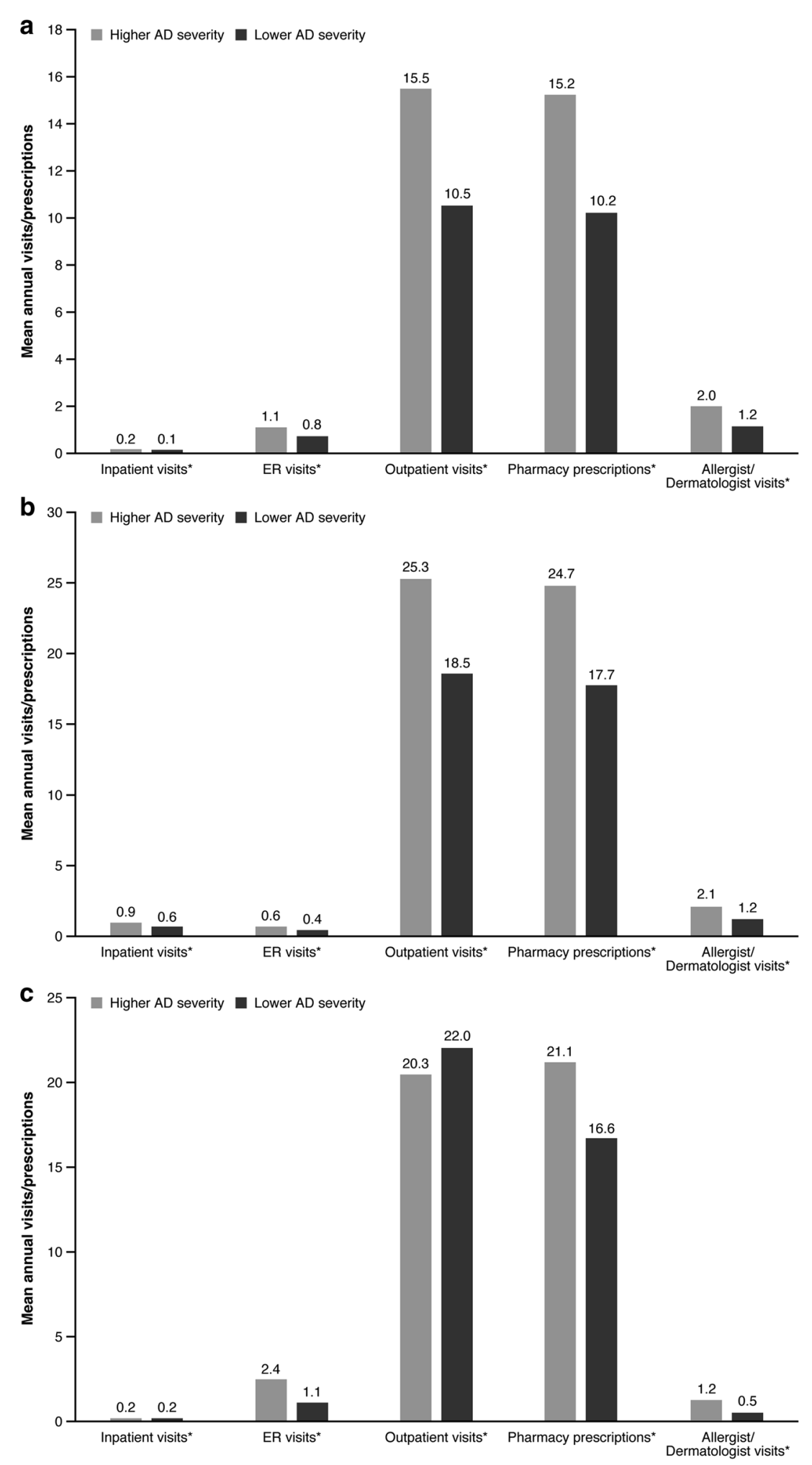

Fig. 6 Mean annual number of healthcare visits per patient stratified by AD disease severity in: a Commercial, b Medicare, and c Medi-Cal populations. ${ }^{*} P<0.05$. Variables non-AD-related comorbidities, and region (Commercial and Medicare only). $A D$ atopic dermatitis, $E R$ emergency included in the generalized linear model: age, gender, race, room 

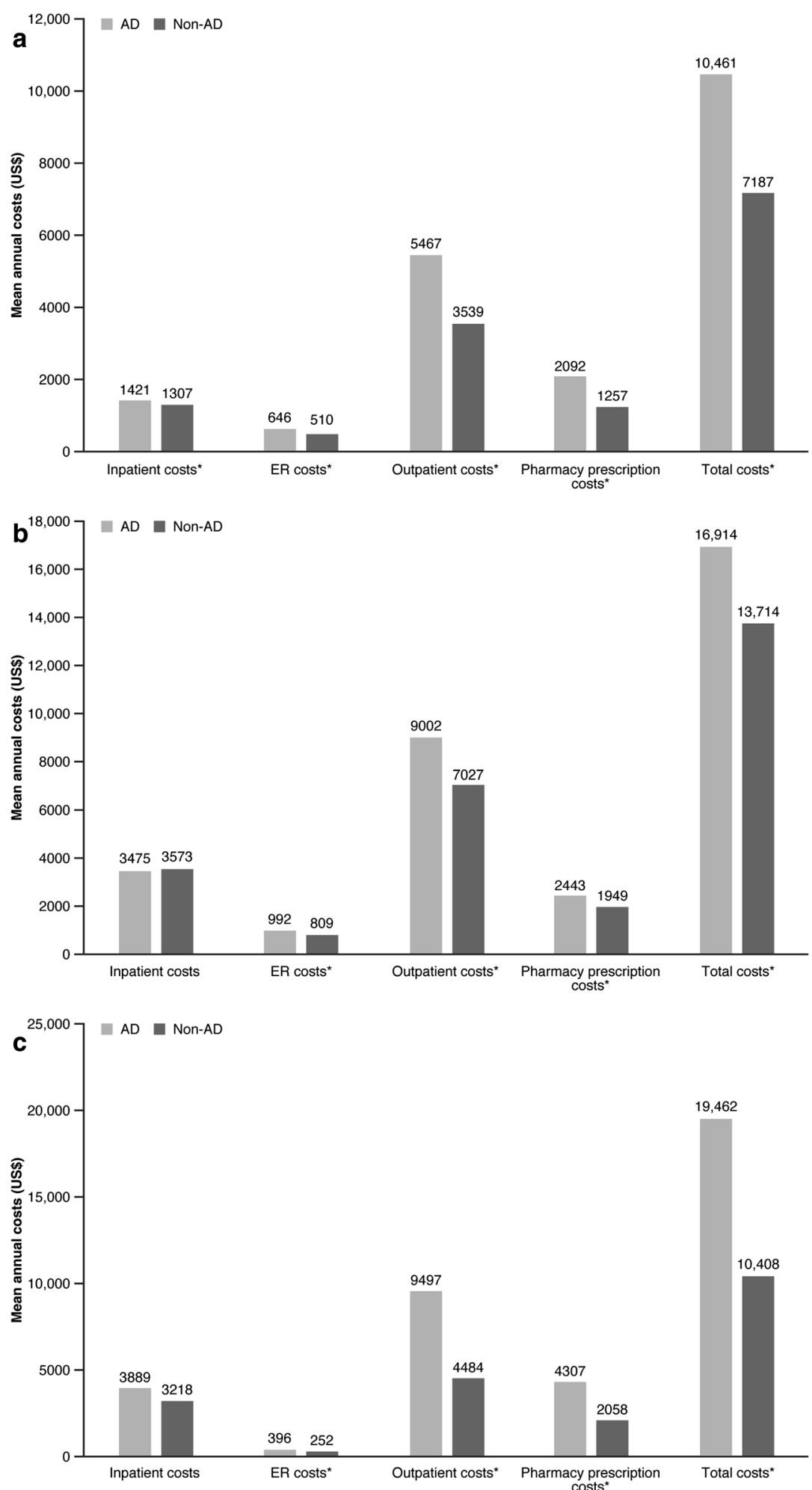

Fig. 7 Adjusted mean annual per-patient healthcare costs in $\mathrm{AD}$ patients and matched non- $\mathrm{AD}$ controls in the: a Commercial, b Medicare, and $\mathbf{c}$ Medi-Cal populations. ${ }^{*} P<0.05$. Variables included in the generalized linear model: age, gender, race, non-AD-related comorbidities, and region (Commercial and Medicare only). $A D$ atopic dermatitis, $E R$ emergency room 

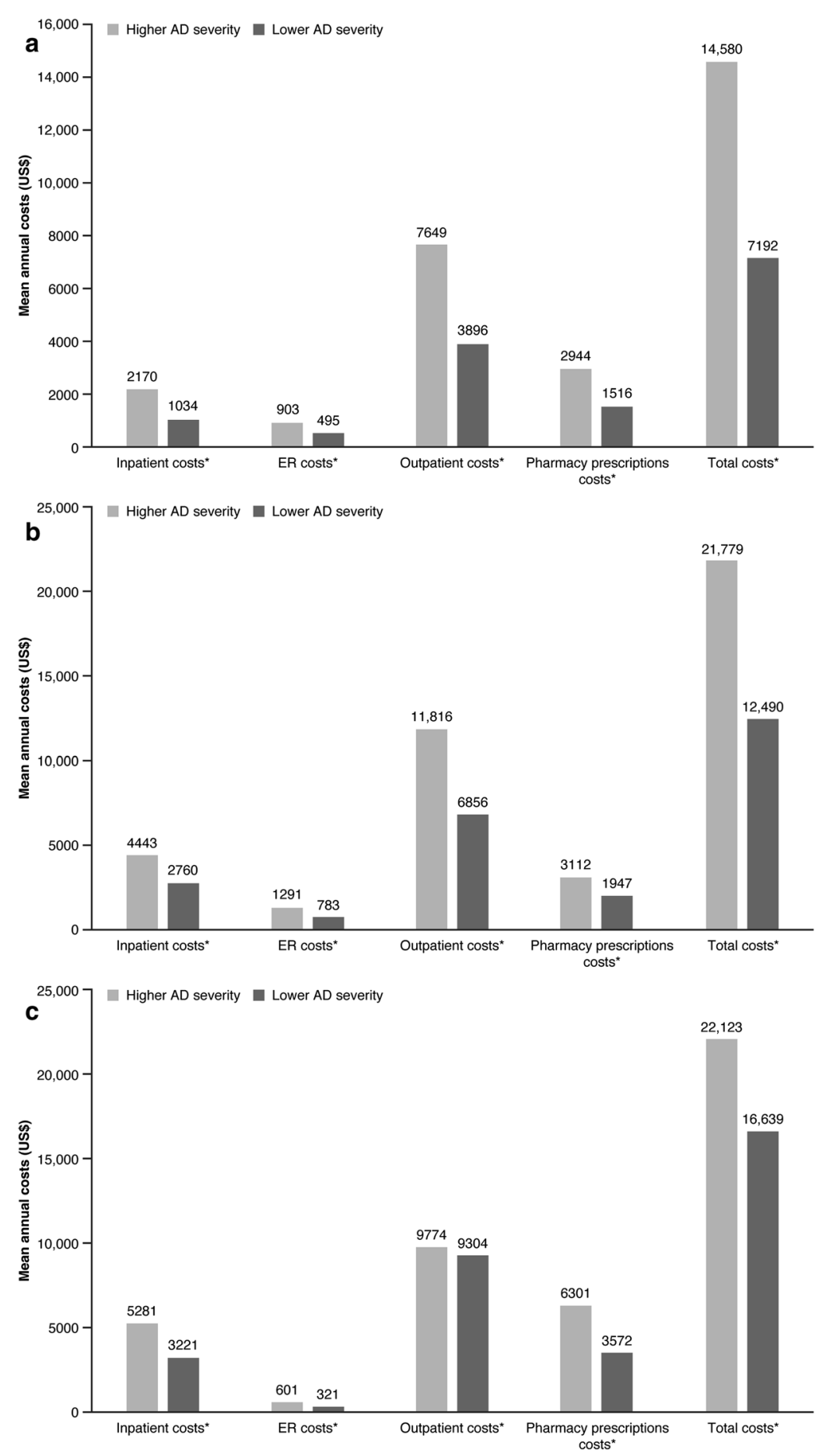

Fig. 8 Adjusted mean annual per-patient healthcare costs stratified by $\mathrm{AD}$ disease severity in: a Commercial, b Medicare, and c Medi-Cal populations. ${ }^{*} P<0.05$. Variables included in the generalized linear model: age, gender, race, non-AD-related comorbidities, and region (Commercial and Medicare only). $A D$ atopic dermatitis, $E R$ emergency room 
moderate-to-severe patients with innovative therapies that reduce their burden to that of lower severity patients. Similar calculations to that described above would yield a mean annual cost offset of US\$7314 per person without considering the costs associated with new therapies. The cost offset is important in research evaluating the cost-effectiveness of a new therapy. A strength of this analysis is its large, geographically and demographically diverse sample from three major healthcare insurance programs in the USA. Thus, the results presented here are likely to be applicable to the general population of US AD adult patients. Controlling for the presence of non-AD-related comorbidities and demographic factors through multivariate analysis allowed us to more precisely estimate the comorbidity burden, HCRU, and costs specifically attributable to $\mathrm{AD}$. The matching of $\mathrm{AD}$ patients and non-AD controls was performed to minimize bias due to demographic characteristics. However, it is possible that some bias remains because of unobservable characteristics. In addition, matching could have obscured the effect of demographic-associated clinical characteristics on comorbidities or disease severity and thus affected outcomes. The key limitation of this analysis is that $\mathrm{AD}$ patients were stratified for disease severity by using treatment as a surrogate measure of severity rather than an objective clinical measure of severity, such as Severity Scoring of Atopic Dermatitis [41] or the Eczema Area and Severity Index [42], because such data are not available in insurance claims databases. Accordingly, we cannot rule out the possibility that variability in treatment patterns between healthcare providers may have introduced bias into the analyses. A further limitation is that only direct healthcare costs were calculated; indirect costs, including the impact of $\mathrm{AD}$ on work productivity and quality of life, were not considered in the cost evaluation.

\section{CONCLUSIONS}

In this large-scale healthcare claims database analysis, patients with $\mathrm{AD}$ had significantly higher comorbidity burden, HCRU, and healthcare costs compared with matched non-AD controls. Higher disease severity was associated with an even greater clinical and economic burden. These data suggest an unmet clinical need for more effective therapies for AD, especially for those patients with higher disease severity.

\section{ACKNOWLEDGEMENTS}

This study and article processing charges were sponsored by Sanofi and Regeneron Pharmaceuticals, Inc. Medical writing support was provided by Fernando Gibson, PhD, Prime, UK, and funded by Sanofi/Regeneron Pharmaceuticals. The authors are responsible for all content and editorial decisions and received no honoraria related to the development of this publication. All authors had full access to all of the data in this study and take complete responsibility for the integrity of the data and accuracy of the data analysis. All named authors meet the International Committee of Medical Journal Editors (ICMJE) criteria for authorship for this manuscript, take responsibility for the integrity of the work as a whole, and have given final approval for the version to be published.

Disclosures. S. Shrestha is an employee of STATinMED Research, which has received research funding from Sanofi/Regeneron Pharmaceuticals Inc. L. Wang is an employee of STATinMED Research, which has received research funding from Sanofi/Regeneron Pharmaceuticals Inc. R. Miao is an employee of and stockholder in Sanofi. H. Yuce is an employee of New York City College of Technology-CUNY, which has received research funding from Sanofi/Regeneron Pharmaceuticals, Inc. J. Chao is an employee of and stockholder in Regeneron Pharmaceuticals, Inc. W. Wei is a former employee of and current stockholder in Sanofi, and is a current employee of Regeneron Pharmaceuticals, Inc.

Compliance with Ethics Guidelines. The study conformed to the Helsinki Declaration of 
1964, as revised in 2013, concerning human and animal rights; Springer's policy concerning informed consent has been followed.

Data Availability. The anonymized datasets analyzed during the current study are available from the corresponding author on reasonable request.

Open Access. This article is distributed under the terms of the Creative Commons Attribution-NonCommercial 4.0 International License (http://creativecommons.org/licenses/ by-nc/4.0/), which permits any noncommercial use, distribution, and reproduction in any medium, provided you give appropriate credit to the original author(s) and the source, provide a link to the Creative Commons license, and indicate if changes were made.

\section{REFERENCES}

1. Weidinger S, Novak N. Atopic dermatitis. Lancet. 2016;387:1109-22.

2. Garmhausen D, Hagemann $\mathrm{T}$, Bieber $\mathrm{T}$, et al. Characterization of different courses of atopic dermatitis in adolescent and adult patients. Allergy. 2013;68:498-506.

3. Illi S, von Mutius E, Lau S, et al. The natural course of atopic dermatitis from birth to age 7 years and the association with asthma. J Allergy Clin Immunol. 2004;113:925-31.

4. Peters AS, Kellberger J, Vogelberg C, et al. Prediction of the incidence, recurrence, and persistence of atopic dermatitis in adolescence: a prospective cohort study. J Allergy Clin Immunol. 2010;126:590-5.

5. Margolis JS, Abuabara K, Bilker W, Hoffstad O, Margolis DJ. Persistence of mild to moderate atopic dermatitis. JAMA Dermatol. 2014;150:593-600.

6. Napolitano M, Megna M, Patruno C, et al. Adult atopic dermatitis: a review. G Ital Dermatol Venereol. 2016;151:403-11.

7. Bannister MJ, Freeman S. Adult-onset atopic dermatitis. Australas J Dermatol. 2000;41:225-8.

8. Kanwar AJ. Adult-onset atopic dermatitis. Indian J Dermatol. 2016;61:662-3.
9. Ozkaya E. Adult-onset atopic dermatitis. J Am Acad Dermatol. 2005;52:579-82.

10. Silverberg JI, Hanifin JM. Adult eczema prevalence and associations with asthma and other health and demographic factors: a US population-based study. J Allergy Clin Immunol. 2013;132:1132-8.

11. Eichenfield LF, Tom WL, Berger TG, et al. Guidelines of care for the management of atopic dermatitis: section 2. Management and treatment of atopic dermatitis with topical therapies. J Am Acad Dermatol. 2014;71:116-32.

12. Eichenfield LF, Tom WL, Chamlin SL, et al. Guidelines of care for the management of atopic dermatitis: section 1. Diagnosis and assessment of atopic dermatitis. J Am Acad Dermatol. 2014;70:338-51.

13. Sidbury R, Davis DM, Cohen DE, et al. Guidelines of care for the management of atopic dermatitis: section 3. Management and treatment with phototherapy and systemic agents. J Am Acad Dermatol. 2014;71:327-49.

14. Langenbruch A, Radtke M, Franzke N, et al. Quality of health care of atopic eczema in Germany: results of the national health care study AtopicHealth. J Eur Acad Dermatol Venereol. 2014;28:719-26.

15. Zeppa L, Bellini V, Lisi P. Atopic dermatitis in adults. Dermatitis. 2011;22:40-6.

16. Kim JP, Chao LX, Simpson EL, Silverberg JI. Persistence of atopic dermatitis (AD): a systematic review and meta-analysis. J Am Acad Dermatol. 2016;75:681-7.

17. The World Allergy Organization (WAO). The WAO white book on allergy: 2013 update. Milwaukee: World Allergy Organization; 2013.

18. Adelphi Real World. Adelphi disease-specific program 2014-2015 [Data on file].

19. Roekevisch E, Spuls PI, Kuester D, Limpens J, Schmitt J. Efficacy and safety of systemic treatments for moderate-to-severe atopic dermatitis: a systematic review. J Allergy Clin Immunol. 2014;133:429-38.

20. Dalgard FJ, Gieler U, Tomas-Aragones L, et al. The psychological burden of skin diseases: a cross-sectional multicenter study among dermatological out-patients in 13 European countries. J Invest Dermatol. 2015;135:984-91.

21. Simpson EL, Bieber T, Eckert L, et al. Patient burden of moderate to severe atopic dermatitis (AD): insights from a phase $2 \mathrm{~b}$ clinical trial of dupilumab in adults. J Am Acad Dermatol. 2016;74:491-8. 
22. Yano C, Saeki H, Ishiji T, et al. Impact of disease severity on work productivity and activity impairment in Japanese patients with atopic dermatitis. J Dermatol. 2013;40:736-9.

23. Whiteley J, Emir B, Seitzman R, Makinson G. The burden of atopic dermatitis in US adults: results from the 2013 National Health and Wellness Survey. Curr Med Res Opin. 2016;32:1-7.

24. Yu SH, Attarian H, Zee P, Silverberg JI. Burden of sleep and fatigue in US adults with atopic dermatitis. Dermatitis. 2016;27:50-8.

25. Charlson M, Szatrowski TP, Peterson J, Gold J. Validation of a combined comorbidity index. J Clin Epidemiol. 1994;47:1245-51.

26. Charlson ME, Pompei P, Ales KL, MacKenzie CR. A new method of classifying prognostic comorbidity in longitudinal studies: development and validation. J Chronic Dis. 1987;40:373-83.

27. Deyo RA, Cherkin DC, Ciol MA. Adapting a clinical comorbidity index for use with ICD-9-CM administrative databases. J Clin Epidemiol. 1992;45:613-9.

28. Andersen YM, Egeberg A, Gislason GH, Skov L, Thyssen JP. Autoimmune diseases in adults with atopic dermatitis. J Am Acad Dermatol. 2017;76:274-80.e1.

29. Simpson EL. Comorbidity in atopic dermatitis. Curr Dermatol Rep. 2012;1:29-38.

30. Boguniewicz M, Leung DY. Atopic dermatitis: a disease of altered skin barrier and immune dysregulation. Immunol Rev. 2011;242:233-46.

31. Leung DY, Guttman-Yassky E. Deciphering the complexities of atopic dermatitis: shifting paradigms in treatment approaches. J Allergy Clin Immunol. 2014;134:769-79.

32. Dharmage SC, Lowe AJ, Matheson MC, et al. Atopic dermatitis and the atopic march revisited. Allergy. 2014;69:17-27.

33. Vanderpuye-Orgle J, Zhao Y, Lu J, et al. Evaluating the economic burden of psoriasis in the United States. J Am Acad Dermatol. 2015;72:961-7.
34. Ellis CN, Drake LA, Prendergast MM, et al. Cost of atopic dermatitis and eczema in the United States. J Am Acad Dermatol. 2002;46:361-70.

35. Fowler JF, Duh MS, Rovba L, et al. The direct and indirect cost burden of atopic dermatitis: an employer-payer perspective. Manag Care Interface. 2007;20:26-32.

36. Fivenson D, Arnold RJ, Kaniecki DJ, et al. The effect of atopic dermatitis on total burden of illness and quality of life on adults and children in a large managed care organization. J Manag Care Pharm. 2002;8:333-42.

37. Carroll CL, Balkrishnan R, Feldman SR, Fleischer AB Jr, Manuel JC. The burden of atopic dermatitis: impact on the patient, family, and society. Pediatr Dermatol. 2005;22:192-9.

38. The Henry J. Kaiser Family Foundation. Health insurance coverage of adults 19-64. http://kff.org/ other/state-indicator/adults-19-64/?currentTime frame $=0$ \&sortModel $=\% 7 \mathrm{~B} \% 22$ colld $\% 22: \% 22$ Loca tion $\% 22, \% 22$ sort $\% 22: \% 22$ asc $\% 22 \% 7 D$. Accessed Mar 2017.

39. Optum $^{\mathrm{TM}}$. Clinoformatics ${ }^{\mathrm{TM}}$ Data Mart 2014 [Data on file].

40. Wei W, Anderson P, Gadkari A, et al. Extent and consequences of inadequate disease control among adults with a history of moderate-to-severe atopic dermatitis. In: Poster presented at the American Academy of Dermatology 74th Annual Meeting, March 4-8, 2016, Washington DC.

41. European Task Force on Atopic Dermatitis. Severity scoring of atopic dermatitis: the SCORAD index. Consensus report of the European Task Force on Atopic Dermatitis. Dermatology. 1993;186:23-31.

42. Hanifin JM, Thurston M, Omoto $M$, et al. The eczema area and severity index (EASI): assessment of reliability in atopic dermatitis. EASI Evaluator Group. Exp Dermatol. 2001;10:11-8. 